\title{
Multilevel Amplitude Regeneration of PAM-4 Signals using a Nonlinear Optical Loop Mirror
}

\author{
Feng Wen(1,2), Christos P. Tsekrekos(1), Xingyu Zhou(2), Yong Geng(2), Baojian Wu(2), Kun Qiu(2), \\ Sergei K. Turitsyn ${ }^{(1)}$ and Stylianos Sygletos ${ }^{(1)}$ \\ (1) Aston Institute of Photonic Technologies, Aston University, B4 7ET Birmingham, UK, \\ f.wen@aston.ac.uk \\ (2) Key Lab of Optical Fiber Sensing and Communication Networks, Ministry of Education, University of \\ Electronic Science and Technology of China, 611731 Chengdu, China
}

\begin{abstract}
Multilevel amplitude regeneration of return-to-zero PAM-4 signals is experimentally demonstrated using a single NOLM unit. The noise suppression capability at each amplitude level is characterized, and $Q^{2}$-factor improvement of $0.92 d B$ is achieved by optimizing input signal power and distortion strength.
\end{abstract}

\section{Introduction}

Advanced modulation formats hold the key to unlock the transmission capacity of fibre communication systems ${ }^{1}$. However, when moving to high constellation orders, signals become more sensitive to transmission impairments that originate either from amplified spontaneous emission noise (ASE) or Kerr nonlinearity. All-optical signal regeneration is seen as a promising and power efficient way to suppress the impact of these effects and improve the quality of the transmitted signal ${ }^{2}$.

Suppression of signal distortion on multiple amplitude or phase levels imposes a significant challenge in the design of the corresponding regenerator sub-system. Using an in-line (i.e. black-box) phase sensitive amplifier, phase noise suppression on 4-levels has been demonstrated enabling the regeneration of QPSK signals ${ }^{3}$. On the other hand, amplitude regeneration has been achieved only for up to two levels using a nonlinear optical loop mirror (NOLM) ${ }^{4}$. Although the potential of the NOLM to support higher number of regenerative levels is known in theory, when pure Kerr-effect based operation is assumed ${ }^{5}$, stimulated Brillouin scattering effects (SBS) have prevented such performance in real experiments.

In this paper we demonstrate, for the first time, the suppression of amplitude distortion simultaneously on four levels and the regeneration of pulse amplitude modulated four signals (PAM-4). This was achieved through the use of short (i.e. 10ps) return-to-zero (RZ) signal pulses, of high peak power, along with a strained aluminous-silicate highly nonlinear fibre (HNLF), which enabled substantial increase of the effective SBS threshold. We note that the scheme can be compatible with current nonreturn-to-zero (NRZ)-based highly spectral efficient transmission systems, through additional NRZ-to-RZ and RZ-to-NRZ conversion circuits. The NRZ-to-RZ conversion can be achieved through an all-optical sampling gate ${ }^{6}$ at the input of the regenerator, whereas the inverse process with simple optical filtering ${ }^{7}$ at the corresponding output. The noise suppression performance of each regenerative level has been carefully characterized and good agreement with theoretical predictions has been found. The signal quality measurements on the PAM-4 showed a $\mathrm{Q}^{2}$-factor improvement of $0.92 \mathrm{~dB}$ compared to the back-to-back case. We believe that the obtained results prove the potential of NOLMs for the all-optical regeneration of advanced modulation formats.

\section{Experimental setup}

The experimental setup of the PAM4 regenerator is demonstrated in Fig. 1. A pulse pattern generator (PPG) triggered by a $10 \mathrm{GHz}$ radio frequency (RF) synthesizer generated a

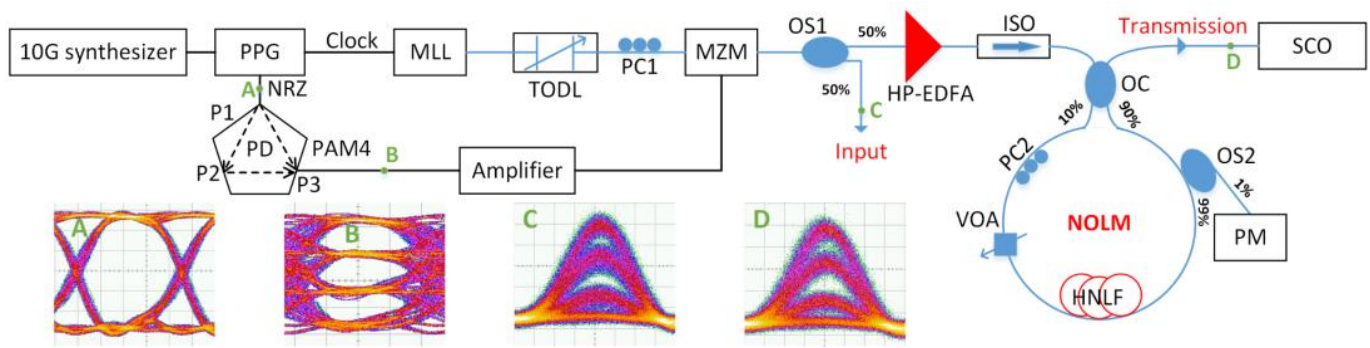

Fig. 1: Experimental setup of PAM4 regeneration in a single NOLM unit 
pseudorandom binary sequence (PRBS) nonreturn-to-zero signal with the length of $2^{31}-1$ and an electronic clock. The NRZ signal was then fed into a power divider (PD) that had a short connected port P2. Combing the split signal from $\mathrm{P} 1$ and the reflected signal from P2 an NRZPAM4 electrical signal was obtained at the output port P3, which was used for the driving of a MachZehnder optical modulator (MZM). The input of the MZM was an optical clock signal of 10ps pulses emitted by a fibre mode-locked laser $(\mathrm{MLL})$ at $1550 \mathrm{~nm}$. A polarization controller (PC) and the tuneable delay line (TODL) helped for the fine signal alignment. Before entering the NOLM unit, the signal power was amplified by a highpower Erbium-doped fibre amplifier (HP-EDFA), while the use of an optical isolator (ISO) prevented unwanted back-propagation of the light to the transmitter.

The NOLM was assembled using a 90:10 optical coupler (OC), a polarization controller, a variable optic attenuator (VOA), a 606m-length strained HNLF and a 99:1 optical splitter. The nonlinear coefficient of the strained HNLF was $7 \mathrm{~W}^{-1} / \mathrm{km}$ and the total fibre loss was $12 \mathrm{~dB}$. The exact input power into the NOLM unit was measured by a power meter (PM) at the $1 \%$ output port of the OS. At the transmission port, the regenerated signal was detected by a $32 \mathrm{GHz}$ optical photodiode, and the signal quality was calculated using the sampling data from the oscilloscope. Eye diagrams of electronic NRZ and NRZ-PAM4, input and regenerated RZPAM4 signals were shown in subfigures $A$ to $D$.

\section{Results and discussions}

Firstly, the power transfer function (PTF) and its slope curves were measured to identify the locations and the range of the regenerative regions. For this an un-modulated clock signal was launched directly into the NOLM and by sweeping the average power of the HP-EDFA we measured the average power at the output of the transmission port. The slope curve has been extrapolated directly from the power transfer function curve. The results of these measurements are depicted in Fig. 2. Four power plateau regions were measured, defined by slope values less than one. The regions were located at 22.5, 25.77, 27.61 and $28.9 \mathrm{dBm}$ power points. We notice that the plateau width decreases when going to higher power levels. This means that the amount of amplitude distortion that can be handled at higher levels also reduces. On the other hand, as the slope values become smaller the noise suppression within these regions becomes more efficient. In the same figure we have plotted the theoretical transfer function and slope curves using the model of [5]. Excellent agreement between theory and experiment is observed.

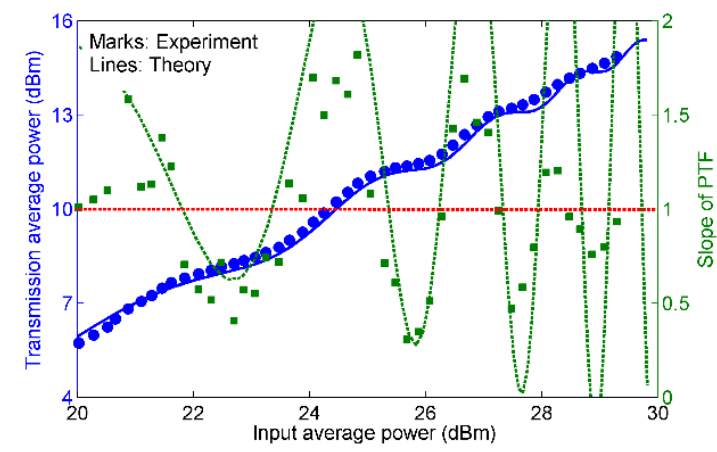

Fig. 2: PTF and its slope of the transmission response

Subsequently, we modulated the clock signal with a PRBS (231-1) amplitude distortion and we measured the achieved noise suppression as a function of the launched optical power. Fig. 3 depicts the peak-to-peak variation of the distortion normalized to the mean peak power of the clock pulses at the input and output of the regenerator. In all measurements the received signal power was kept fixed at $-10 \mathrm{dBm}$. We notice that following the slope curve of the regenerator's transfer function the output distortion was enhanced or suppressed. The first two suppression regions were clearly observed due to their broader width, but the boundary between the third and fourth region was less clear due to their significantly smaller range. Therefore, it was more practical to combine the last two regions in one and consider in total the existence of three regenerative regions, $A, B$ and $C$ located at $23.07,26.01,28.08 \mathrm{dBm}$, respectively, see Fig. 3.

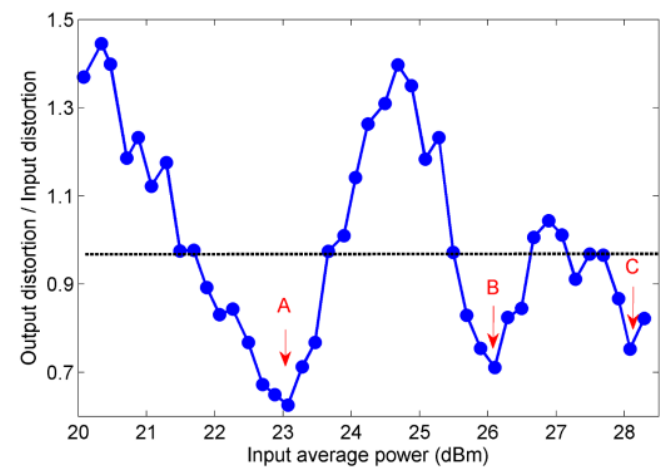

Fig. 3: Amplitude noise transfer with input powers

The next step was to investigate more thoroughly the achieved suppression in each one of the three regenerative regions for different amounts of introduced amplitude distortion. Fig. 4 depicts the normalized amplitude distortion at the output as a function of the normalized input distortion. The points below the diagonal line designate that amplitude suppression has been achieved. Also in this case it is obvious that more 
noise could be handled in the first region. We also notice that for a normalized amplitude distortion range between 0.276 and 0.62 we could have noise suppression simultaneously on the three regions. In a way, this range defines the tolerance of the regenerator to the input distortion.

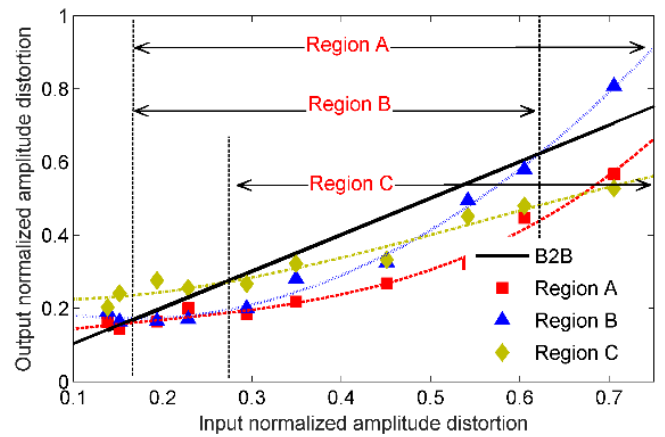

Fig. 4: Noise suppression capability for each regenerative amplitude levels

Finally, we tuned the TODL to introduce the amplitude noise by sampling different part of electronic NRZ-PAM4, and then put the noisy RZPAM4 signal into the multilevel regenerator. By adjusting the average power level at the input of the NOLM an optimum operating point could be identified at $24.08 \mathrm{dBm}$, which could provide an overall $\mathrm{Q}^{2}$-factor improvement of $0.88 \mathrm{~dB}$, see Fig. 5. The $\mathrm{Q}^{2}$-factor was calculated from the error vector magnitude $(E V M), Q^{2}=10 \log _{10}\left(1 / E V^{2}\right)^{8}$. At the optimum input power point we characterized the regenerative performance as a function if the input signal distortion, see Fig. 6. A maximum $\Delta \mathrm{Q}^{2}$ improvement of $0.92 \mathrm{~dB}$ was obtained when the quality of the input signal was characterized by $20.5 \mathrm{~dB}$ in the $\mathrm{Q}^{2}$. The $2^{\text {nd }}$, $3^{\text {rd }}$ and $4^{\text {th }}$ levels of the PAM signal fell into the corresponding characterized plateau regions $A$, $B$ and $C$ of the regenerator. Typical power histograms, also depicted in Fig. 6 confirm this conclusion and the noise suppression achieved at these levels. The eye linearity defined by the ratio of the largest to smallest mean voltage swing between symbols ${ }^{9}$ was slightly changed by $3.74 \%$ compared to that of input signal, which maintained the signal linearity after the regeneration.

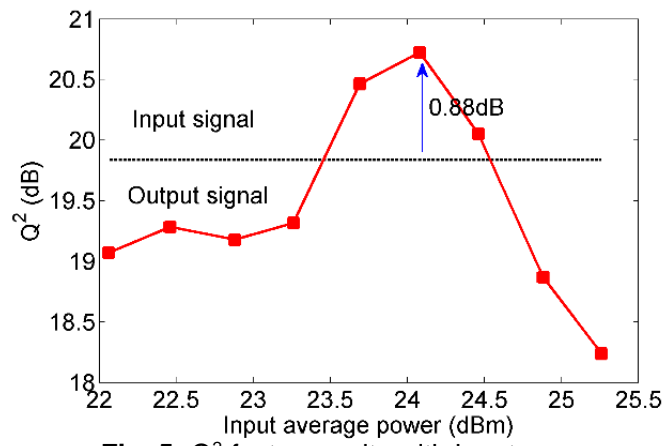

Fig. 5: $Q^{2}$-factor results with input powers

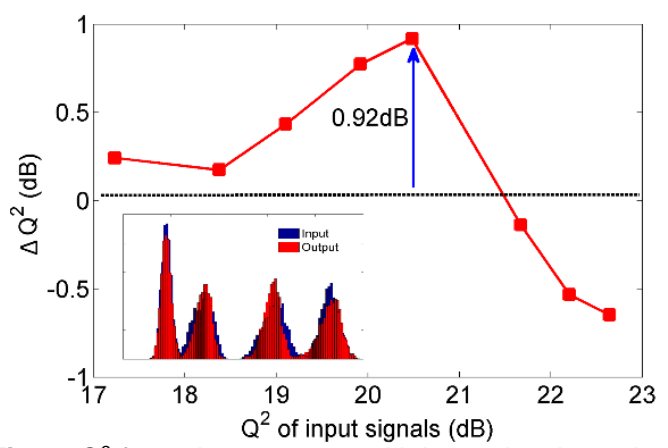

Fig. 6: $Q^{2}$-factor improvement with input signals, and power histograms before and after the regeneration

\section{Conclusions}

Multilevel amplitude regeneration has been investigated in a NOLM-based all-optical interferometer. Multiple power plateau regions were obtained from the transmission response, and operation ranges for each amplitude levels were identified by the slope of PTF curves. The noise suppression capability of the scheme was discussed for each regenerative level, and amplitude regeneration for RZ-PAM4 signals was achieved, by optimizing the input power operating conditions, with a $\mathrm{Q}^{2}$-factor improvement of $0.92 \mathrm{~dB}$.

\section{Acknowledgements}

The authors would like to thank the Tyndall National Institute for the loan of the highly nonlinear fibre, and Ian Phillips, Marc Srephens and Filipe Ferreira for valuable discussions. This work has been supported by the EPSRC project UNLOC (EP/J017582/1), the Marie Sklodowska-Curie Action (701770INNOVATION), the National Natural Science Foundation of China $(61505021,61671108)$, and the Program for Changjiang Scholars and Innovative Research Team in Universities of China (IRT1218), 111 Project (B14039).

\section{References}

[1] R.-J. Essiambre et al., Proc. IEEE, Vol. 100, no. 5, p. 1035 (2012).

[2] M. Sorokina, Opt. Lett., Vol. 39, no. 8, p. 2499 (2014).

[3] J. Kakande et al., Proc. ECOC, PD 3.3, Torino (2010).

[4] T. Roethlingshoefer et al., Opt. Express, Vol. 22, no. 22, p. 27077 (2014).

[5] F. Wen et al, Accepted by ICTON 2017, Girona (2017).

[6] S. J. Fabbri et al., J. Lightwave Technol., Vol. 33, no. 7, p. 1351 (2015).

[7] A. Perentos et al., Opt. Express, Vol. 24, no. 15, p. 16649 (2016).

[8] R Schmogrow et al., IEEE Photon. Technol. Lett. Vol 24, no. 1, pp 61-62, 2011.

[9] Tektronix, "PAM4 Signaling in High Speed Serial Technology: Test, Analysis, and Debug," Application note. http://tmsinc.co.kr/media/uploads/tek/application/[dpo70 000sx]pam4_signaling_in_high_speed_serial_technolog y_test_analysis_and_debug.pdf 